\title{
Enhanced whole exome sequencing by higher DNA insert lengths
}

\author{
Claudia Pommerenke ${ }^{1 *}$, Robert Geffers ${ }^{2}$, Boyke Bunk¹, Sabin Bhuju², Sonja Eberth, Hans G. Drexler ${ }^{1}$ \\ and Hilmar Quentmeier ${ }^{1}$
}

\begin{abstract}
Background: Whole exome sequencing (WES) has been proven to serve as a valuable basis for various applications such as variant calling and copy number variation (CNV) analyses. For those analyses the read coverage should be optimally balanced throughout protein coding regions at sufficient read depth. Unfortunately, WES is known for its uneven coverage within coding regions due to GC-rich regions or off-target enrichment.

Results: In order to examine the irregularities of WES within genes, we applied Agilent SureSelectXT exome capture on human samples and sequenced these via lllumina in $2 \times 101$ paired-end mode. As we suspected the sequenced insert length to be crucial in the uneven coverage of exome captured samples, we sheared 12 genomic DNA samples to two different DNA insert size lengths, namely 130 and $170 \mathrm{bp}$. Interestingly, although mean coverages of target regions were clearly higher in samples of $130 \mathrm{bp}$ insert length, the level of evenness was more pronounced in $170 \mathrm{bp}$ samples. Moreover, merging overlapping paired-end reads revealed a positive effect on evenness indicating overlapping reads as another reason for the unevenness.

In addition, mutation analysis on a subset of the samples was performed. In these isogenic subclones, the false negative rate in the $130 \mathrm{bp}$ samples was almost double to that in the $170 \mathrm{bp}$ samples. Visual inspection of the discarded mutation sites exposed low coverages at the sites flanked by high amplitudes of coverage depth.

Conclusions: Producing longer insert reads could be a good strategy to achieve better uniform read coverage in coding regions and hereby enhancing the effective sequencing yield to provide an improved basis for further variant calling and CNV analyses.
\end{abstract}

Keywords: Whole exome sequencing, DNA insert size, Read coverage, Evenness score, Variant calling

\section{Background}

During the past years whole exome sequencing (WES) has gained much popularity in research and diagnostics, as focussing on protein-coding regions reduces sequencing costs compared to whole genome sequencing (WGS) [1-4]. Concentrating on exonic regions minimises the sequencing target area of the human genome with about $3 \mathrm{Gbp}$ to less than $2 \%[3,4]$. There is a broad application area of WES such as variant calling [3-5] and analysis of copy number variations (CNV) $[6,7]$ demonstrating its general usefulness in the genomic field.

*Correspondence: claudia.pommerenke@dsmz.de

1 Leibniz Institute DSMZ-German Collection of Microorganisms and Cell

Cultures, Inhoffenstrasse 7B, 38124 Braunschweig, Germany

Full list of author information is available at the end of the article
In terms of quality validation, many research efforts were focussing on the comparison of different exome enrichment platforms [8-11] and their performance to WGS techniques [12, 13]. Apparently, one recurrent bias appearing with WES is its inhomogeneous coverage across targeted protein-coding regions, which is suggested to be resolved by increasing read depth. However, increasing depth for WES would also place the economic costs for the alternative WGS in similar range to WES $[12,13]$. For example, in one study the exome coverage of $\geq 20 \times$ needed an average of $160 \times$ with WES, whereas WGS was sufficient with 44-56× [12].

In the midst of this debate, we would like to propose a new aspect to the technical side of WES. During standard WES $(2 \times 101$ paired-end sequencing $)$ of our cancer cell lines, we observed a strong irregular distribution of 
read coverage along exons, which had a size of $\geq 1 \mathrm{kbp}$. In the following, the genomic DNA fraction sequenced as paired-end reads and flanked by illumina adapters is denominated as insert, in order to confine these to sequencing library fragments including illumina adapters. Calculation of the original DNA insert size revealed that the genomic DNA insert had a peak size of $130 \mathrm{bp}$. This prompted us to consider, whether longer purified DNA inserts might improve the evenness of read coverage. In the past, DNA insert lengths of 200-250 bp for $2 \times 90$ paired-end reads were mentioned to contribute to library optimisation, nonetheless, results were not shown [8]. Additionally, different fragmentation techniques improved coverage, yet DNA insert length were indicated for sonication (161 bp) but not for enzymatic fragmentation [10]. In another study short inserts of 100$200 \mathrm{bp}$ are suggested instead of 500-800 bp [1], however, at that time the development of exome capture design just started and more importantly, very short single reads of $26 \mathrm{bp}$ were analysed. Similarly, short reads of 35 and $50 \mathrm{bp}$ were sequenced for a further publication, in which short insert sizes of $120 \mathrm{bp}$ are recommended given the short median length of $120 \mathrm{bp}$ of human exons [14]. Apparently, no specific study on the effect of DNA insert sizes to paired-end sequencing has been published to date.

Therefore, we tested in this study, whether the genomic DNA insert length influences the uniformity of read coverage within targeted regions. Beside samples with 130 bp peak insert length, a sample group with 170 bp peak inserts was produced and validated. The evenness score [14] was applied as metric for assessing the effectiveness of target region coverage. Altogether, we would like to provide a short technical note on the effect of DNA insert length on the evenness of coverage for paired-end sequenced reads.

\section{Results and discussion}

Production of WES libraries with two different insert sizes

For exploring the effect of different genomic DNA insert lengths on the uniformity of coverage in whole exome sequencing (WES), we varied the DNA shearing by acoustic fragmentation (Covaris). Two DNA inserts groups of six samples each were yielded with approximately 130 and 170 bp length (Fig. 1a+b). Exome enrichment was conducted with Agilent SureSelectXT All Exon v5+UTR/v5 and sequencing produced $18-54$ million reads per sample (see Table 1). After trimming, removing PCR duplicates and mapping the reads to the human GENCODE genome v21, manual inspection of alignments to many exons exceeding $1 \mathrm{kbp}$ indicated large amplitudes of coverage for $130 \mathrm{bp}$ compared to $170 \mathrm{bp}$ samples (Fig. 1c). This observation, that longer inserts might compensate for "mountain-valley" profiles in coverage, encouraged us to examine this in detail.
Since the mean DNA insert peak for each sample group with 130 and $170 \mathrm{bp}$, respectively, was smaller than the resulting paired-end sequenced reads of $2 \times 101 \mathrm{bp}$, a high percentage of paired-end reads contained overlapping sequences. As these overlaps did not contain further information for e.g. variant calling and $\mathrm{CNV}$ analysis, pairedend reads were joined where overlapping sequences were found and aligned to the human genome. Trimming, read mapping and joining statistics are summarised in Table 2 and Phred quality scores for the sequencing cycles are visualised in Fig. 1d. Nearly all sequenced bases for further analyses had quality scores $\geq 30$.

In the following sections unmerged and merged sequences are compared along with contrasting 130 versus 170 bp insert results.

\section{Differences in exome capture and read depth}

We applied two different exome enrichment kits in this study, namely Agilent SureSelectXT v5+UTR and v5 on the 130 bp genomic DNA insert and 170 bp group, respectively (see Table 1). The genomic target region both kits are covering were nearly identical except for the UTR stretches which were exclusively contained in v5+UTR: v5 target regions constitute $68 \%$ of v5+UTR $(75 \mathrm{Mb})$ and $99.9996 \%$ of v5 (50 Mb) were included in v5+UTR (Fig. 2a). Hence, for further comparison the specific target region needed to be considered.

Although aiming to adapt the read numbers to the respective target regions during the sequencing process, the mean coverage to the target region sizes 75 and 50 Mbp was increased for $130 \mathrm{bp}$ compared to $170 \mathrm{bp}$ inserts (Fig. 2b). However, this difference attenuated when computing coverage means for merged paired-end sequences. Similar results were obtained when calculating the mean coverage of 130 and $170 \mathrm{bp}$ inserts on their respective target regions and on overlapping target regions of $\mathrm{v} 5$ and $\mathrm{v} 5+\mathrm{UTR}$ (Fig. 2c). Intriguingly, concentrating on the fraction in the respective target region at $\geq 10 \times$ read depth revealed a smaller fraction of $130 \mathrm{bp}$ samples covered at that mininum read depth than for 170 bp (Fig. 2d). Low coverages in turn mean impaired mutation detection in these regions, whereas at the same time excessive coverage of target regions in 130 bp samples (Fig. 1c) seems dispensible.

\section{Comparing uniformity of coverage}

As a measure for the skewed distribution of exome captured sequences in the coding regions, the evenness score was calculated allowing for target region and library size correction [14] (Fig. 3). Hereby, after normalising the coverage of each sample to the integral for each coverage curve to 1 (Fig. $3 \mathrm{a}+\mathrm{b}$ ), the area below the curve between 0 and 1 is defined as the evenness score [14]. Bearing in mind that the higher the evenness score, the 

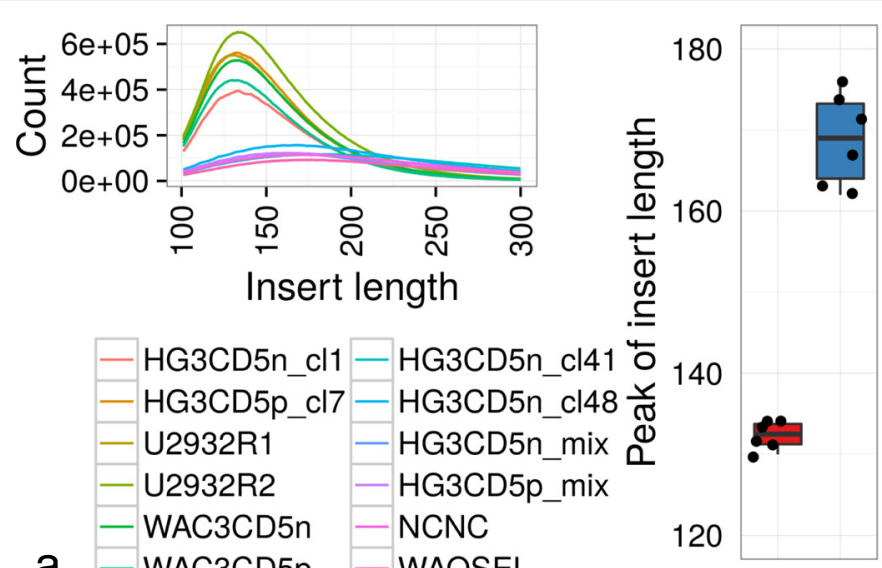

\section{Name assignment \\ 官 130 \\ 官170}
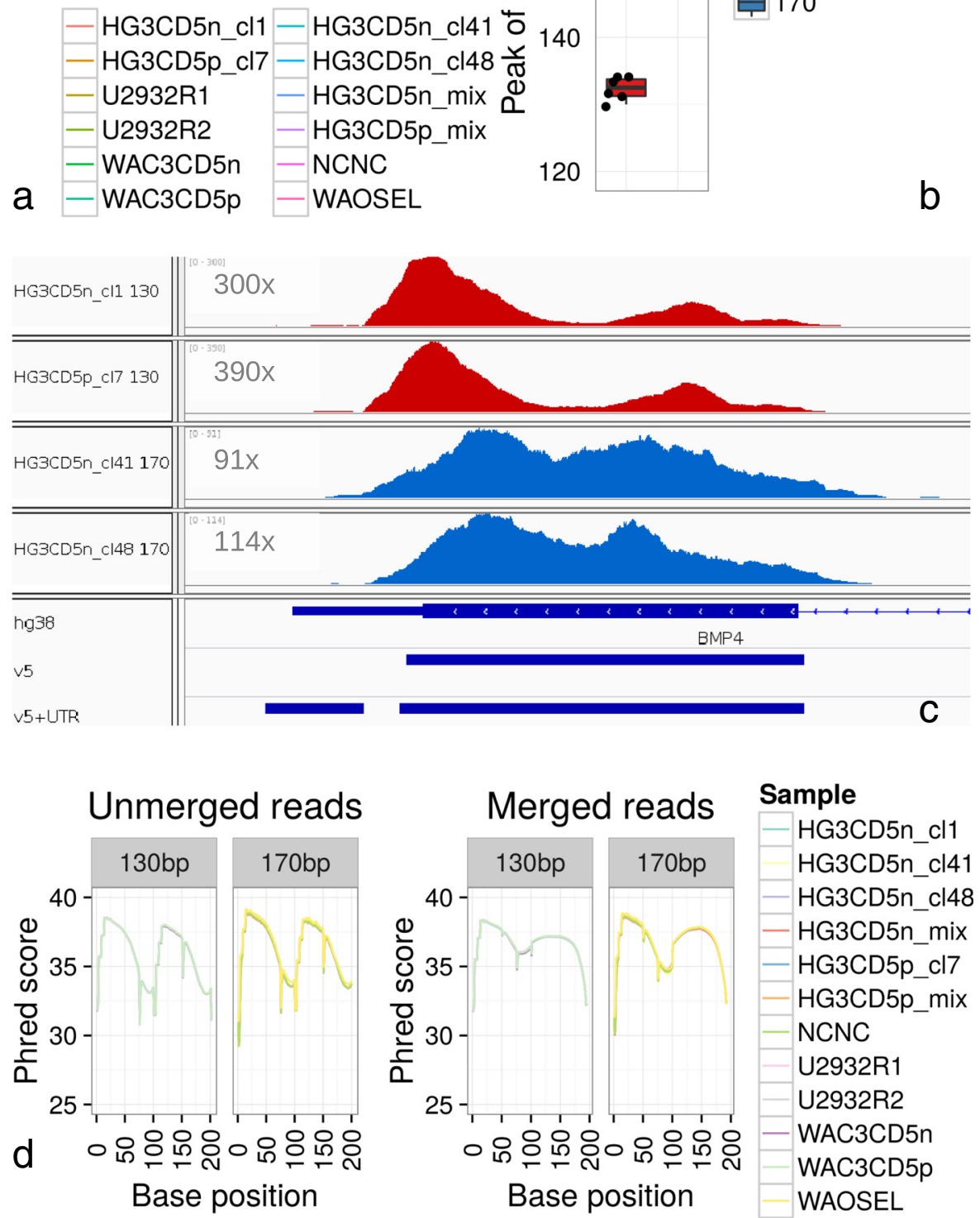

Fig. 1 DNA shearing to 130 and 170 bp fractions before Illumina adapter ligation; sequencing base quality. a DNA insert length distribution per sample. b Peak insert lengths for the two different sample groups. c Alignment histograms for $130 \mathrm{bp}$ insert samples (red) exhibited high amplitudes of coverage within the exon in comparison to $170 \mathrm{bp}$ (blue) as exemplified by this gene BMP4 via IGV. Target regions of Agilent v5 and v5+UTR are given in the last two lines. Please note the $3 \times$ fold higher maximum coverage of $130 \mathrm{bp}$ samples. $\mathbf{d}$ High Phred score quality values for mapped paired-end reads. Base calling quality was high after trimming and mapping to the human genome. As expected, for both reads in forward and reverse direction (1-100 and 101-200 bases) read quality increased during the first 10 cycles and dropped gradually due to de-phasing errors of Illumina's sequencing pipeline. After joining paired-end reads, quality scores improved between $75-125$ cycles, as the best scores were kept while merging. Quality scores were $\geq 30$ throughout nearly all cycles and similar between 130 and 170 bp samples 
Table 1 Portfolio of the samples in this study

\begin{tabular}{lllll}
\hline Sample & Cell line* & $\begin{array}{l}\text { Agilent } \\
\text { SureSelectXT }\end{array}$ & $\begin{array}{l}\text { Insert } \\
\text { length bp }\end{array}$ & $\begin{array}{l}\text { Mio. } \\
\text { Reads }\end{array}$ \\
\hline HG3CD5n_cl1 & HG3 & v5+UTR & 134 & 32.4 \\
HG3CD5p_cl7 & HG3 & v5+UTR & 132 & 44.4 \\
U2932R1 & U-2932 & v5+UTR & 131 & 44.1 \\
U2932R2 & U-2932 & v5+UTR & 133 & 53.8 \\
WAC3CD5n & WA-C3CD5+ & v5+UTR & 134 & 43.3 \\
WAC3CD5p & WA-C3CD5+ & v5+UTR & 130 & 35.9 \\
HG3CD5n_cl41 & HG3 & v5 & 176 & 25.9 \\
HG3CD5n_cl48 & HG3 & v5 & 167 & 27.9 \\
HG3CD5n_mix & HG3 & v5 & 162 & 19.8 \\
HG3CD5p_mix & HG3 & v5 & 163 & 21.8 \\
NCNC & NC-NC & v5 & 171 & 22.2 \\
WAOSEL & WA-OSEL & v5 & 174 & 18.0 \\
\hline
\end{tabular}

${ }^{*}$ All cell lines are held at the DSMZ

more even the coverage, the evenness score for $170 \mathrm{bp}$ insert samples was clearly higher than $130 \mathrm{bp}$ and hence its coverage more even than the 130 bp group (Fig. 3c) despite the higher average coverage of 130 bp (Fig. 2c). Excluding overlapping sequences within the paired-end reads yielded a substantial rise in evenness for both insert groups.

Since using different enrichment kits for 130 and 170 bp samples respectively, the additional UTR target region fraction might be the cause of the unevenness observed in $130 \mathrm{bp}$ inserts. However, the differences in the evenness scores were comparable to the results above when calculated on the common target region of v 5 for both 130 and 170 bp inserts (Fig. 3c). The augmented evenness score for $130 \mathrm{bp}$ inserts in the common coding target regions compared to its v5+UTR target region might even hint on inferior read coverage in the UTRs or inversely enhanced coverage (Fig. 2c) and uniformity for the coding regions.

For WES applying paired-end sequencing, libraries with small genomic DNA insert length produce overlapping sequences. These overlapping bases within one pairedend read provide no extra information on an alternate DNA strand or another allele, since they stem from the identical original genomic DNA sequence. The more overlapping bases within a paired-end read, the more bases remain unused, hence the effectivity to gain coverage shrinks with low insert sizes. Moreover, joining pairedend reads yielded in higher evenness scores particularly for $130 \mathrm{bp}$ inserts (Fig. 3c), thereby showing another negative impact of redundant overlapping sequences.

On the other side, the median size of human exons is 120 bp, thus many bases will map off-target with longer inserts [14], however, the coverage in longer exons would reach higher uniformity and higher minimum depth (Fig. 2) instead of distinct "mountain-valley" coverage silhouette. Increasing coverage depth unfortunately would not yield in proportionally homogeneous coverage (Fig. 2 and [12]). Higher evenness in turn is prerequisite to effectively detect mutations, which is achieved with longer inserts (Fig. 3c). These longer inserts can be produced by a plain technical adjustment in the DNA sample fragmentation step. It may be speculated that peak library sizes of $>200$ bp will result in even better evenness and simultaneously minimise costly overlapping read bases and reduce excess coverages.

\section{Missing mutations in shorter insert samples}

To demonstrate that shorter DNA inserts do increase the false negative rate for mutation analysis, we included four isogenic subclones of the human cell line HG-3. All four HG-3 subclones were sequenced at a comparable depth to

Table 2 Preprocessing statistics

\begin{tabular}{|c|c|c|c|c|c|c|}
\hline \multirow[b]{2}{*}{ Sample } & \multicolumn{2}{|c|}{ Trimming R1 } & \multicolumn{2}{|c|}{ Trimming R2 } & \multirow{2}{*}{$\begin{array}{l}\text { Mapped } \\
\text { reads }\end{array}$} & \multirow{2}{*}{$\begin{array}{l}\text { Joined } \\
\text { reads }\end{array}$} \\
\hline & reads & bases & reads & bases & & \\
\hline HG3CD5n_Cl1 & $19,9 \%$ & 9,0 & $22,4 \%$ & 22,2 & $93,7 \%$ & $80,5 \%$ \\
\hline HG3CD5p_cl7 & $20,0 \%$ & 8,8 & $22,4 \%$ & 22,1 & $93,6 \%$ & $82,4 \%$ \\
\hline U2932R1 & $20,1 \%$ & 8,9 & $22,1 \%$ & 21,8 & $93,5 \%$ & $81,9 \%$ \\
\hline U2932R2 & $20,0 \%$ & 8,9 & $22,6 \%$ & 22,8 & $93,7 \%$ & $80,1 \%$ \\
\hline WAC3CD5n & $22,6 \%$ & 8,9 & $20,0 \%$ & 22,4 & $93,5 \%$ & $80,6 \%$ \\
\hline WAC3CD5p & $20,0 \%$ & 9,0 & $22,4 \%$ & 21,8 & $93,7 \%$ & $81,6 \%$ \\
\hline HG3CD5n_c|41 & $14,6 \%$ & 12,8 & $12,7 \%$ & 43,1 & $90,5 \%$ & $38,0 \%$ \\
\hline HG3CD5n_c|48 & $14,5 \%$ & 12,6 & $12,5 \%$ & 42,7 & $90,3 \%$ & $47,1 \%$ \\
\hline HG3CD5n_mix & $14,3 \%$ & 12,5 & $12,1 \%$ & 41,9 & $90,6 \%$ & $50,8 \%$ \\
\hline HG3CD5p_mix & $13,7 \%$ & 12,3 & $11,9 \%$ & 42,4 & $91,0 \%$ & $46,8 \%$ \\
\hline NCNC & $14,6 \%$ & 12,7 & $12,4 \%$ & 43,7 & $89,2 \%$ & $43,8 \%$ \\
\hline WAOSEL & $13,3 \%$ & 15,9 & $10,3 \%$ & 42,4 & $88,0 \%$ & $44,4 \%$ \\
\hline
\end{tabular}




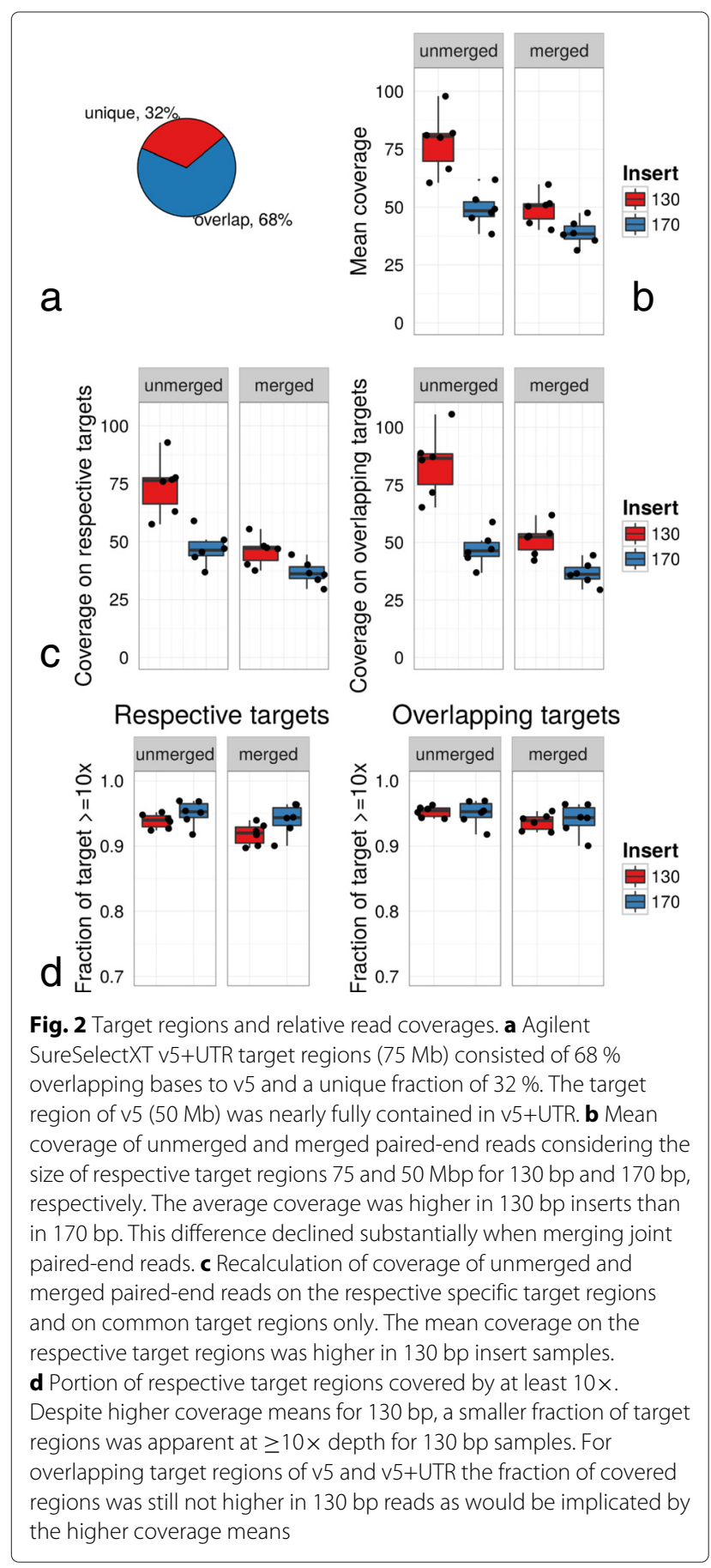

their respective target regions. Of 9087 mutations found in at least one of the subclones, 223 and 193 were missed in the 170 bp samples but nearly twice as many mutations (540 and 375) in $130 \mathrm{bp}$ (Fig. 4a), although coverage was slightly higher in $130 \mathrm{bp}$ inserts for common target coding regions (Fig. 2c).

Manual inspection of 44 selected mutations with a minimum depth $\geq 20 \times$ in both 130 bp peak insert samples and simultaneously $<10 \times$ in $170 \mathrm{bp}$ samples and vice versa delivered mutations in four genes, that were discarded by longer insert samples, but mutations in 20 genes were missed by shorter inserts (e.g. Fig. 4b). Many of the failed mutations by the shorter inserts were found in regions, where amplitudes between maximum and minimum depth were high in the target regions forming a "mountain-valley" pattern as for the mutation in OR5H15 (Fig. 4c). Although this mutation dropped out in the shorter insert samples due to low depth in a "valley", the biallelic nature seen in the longer insert was also true for shorter inserts. Here, higher coverage depth in target regions for $130 \mathrm{bp}$ samples could not improve mutation detection in this gene. Expanding the insert length resulted in mapping more reads to off-target regions of OR5H15, which was more appropriate for this bait design.

The question arose whether the bait-balancing differences between the two exome enrichment kits may account for the skewed coverage of $130 \mathrm{bp}$ compared to $170 \mathrm{bp}$ samples. Bait-balancing is applied for adjusting the number of probes to the binding efficiency of the targeted regions. For OR5H15 as an example all coverage histogram peaks reflected the target regions, which were identical to v5 and v5+UTR (Fig. 4c). An unoptimised bait-balance between the given oligos within this single exon for both insert groups was apparent and the bait-balance of the v5 and v5+UTR kit was similar if not identical. It seems, that the manufacturer's recommended 130-150 bp peak insert fragmentation was insufficient to span the entire annotated exon of OR5H15 on the basis of the target region rather than due to bait-balancing effects of the different capture kits used.

To overcome this technical shortcoming, the baits of the capture assay could be redesigned in closer proximity, or the potential of the current platform could be exploited by applying longer insert sizes. This would be a trade-off between too low as well as excessive read coverages and capturing off-target regions. Since flanking sequences to target regions often reside within annotated gene regions, achieving higher uniform coverage should be prioritised.

\section{Conclusions}

Although widely used, one major drawback of WES is its skewed coverage distribution within the targeted exome. By simply enlarging genomic DNA fragment sizes before exome capturing, the evenness of coverage can be augmented. We think that WES with an average coverage of $80 \mathrm{x}$ in contrast to WGS will remain feasible for smaller studies with limited budget in the next years, therefore any optimisation of this technology is assumed to affect a broad community.

Hence, increasing the DNA insert length maybe even longer than $170 \mathrm{bp}$ will gain better uniform read coverage for WES and thus provide an improved basis for variant calling and $\mathrm{CNV}$ analyses at minimised sequencing costs. 

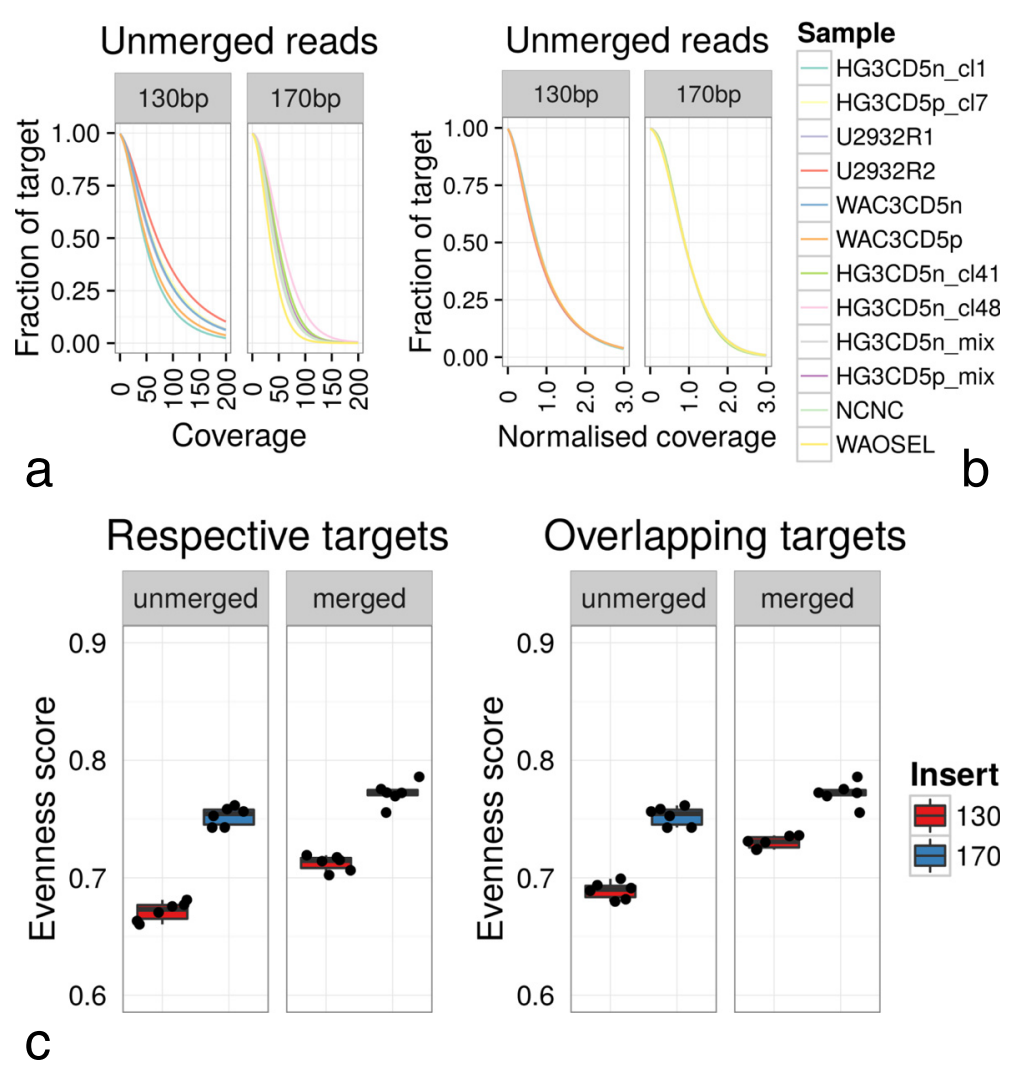

Fig. 3 Evenness between different insert groups and unmerged/merged sequences. Before (a) and after (b) normalisation of coverage to the fraction of respective target regions for unmerged sequences. The complete integral of normalised coverage to the target region is summing up to 1. c The evenness score computed from the area under the curve of unmerged (Fig. 3b) and merged sequences between 0 and 1 normalised coverage. The closer the evenness score is to 1 , the better the uniformity of base coverage. The impact of higher insert length was evident; merged inserts gained top evenness scores regardless of relating to the specific corresponding target region or to overlapping target regions

\section{Methods}

\section{Samples, exome enrichment and sequencing}

A selection of $2 \times 6$ human cancer cell lines (see Table 1 ) was prepared for WES all held by the DSMZ cell line bank (http://www.dsmz.de) and cultured as described previously [15].

Fragmentation of $100 \mathrm{ng}$ purified genomic DNA (gDNA) in $55 \mu$ l Tris-EDTA buffer was done on Covaris S2 and the procedure adjusted to obtain fragments with a peak length of 130 and $170 \mathrm{bp}$, respectively. After library preparation from $100 \mathrm{ng}$ of fragmented gDNA using Agilent SureSelectXT v5 (50 Mb) and v5+UTR (75 Mb), libraries were purified, size validated and prepared for sequencing according to the manufacturer's protocols. Libraries were sequenced on Illumina HiSeq2500 using TruSeq SBS Kit v3-HS $(2 \times 101$, paired-end run). Concentration, quality, fragment sizes of purified genomic DNA (gDNA) and libraries were controlled by Agilent Technologies 2100 Bioanalyzer (Agilent Technologies; Waldbronn, Germany).

\section{Sequence processing, mapping and data analysis}

Before mapping raw reads in fastq format, sequences were trimmed at the ends for low quality $(<\mathrm{Q} 20)$ or adapter contamination by fastq-mcf of the ea-utils toolbox (version 1.1.2-686). Subsequently, trimmed reads were evaluated via FastQC (version 0.11.3, http://www. bioinformatics.babraham.ac.uk/projects/fastqc). For another branch of the pipeline trimmed reads were merged via fastq-join of ea-utils (version 1.1.2-686). Alignments of unmerged trimmed reads and merged reads were carried out with STAR (version 2.4.1d) [16] to the v21/hg38/GRCh38 assembly of the human reference genome. After conversion of sam files to sorted bam files by samtools (version 0.1.19), PCR duplicates were removed via Picard tools (version 1.121, http://picard. sourceforge.net).

For visual inspection of alignments the IGV was applied [17]. Coverage was calculated by bedtools2 (version 2.19.1) based on the target region design Agilent provided at https://earray.chem.agilent.com/suredesign for 


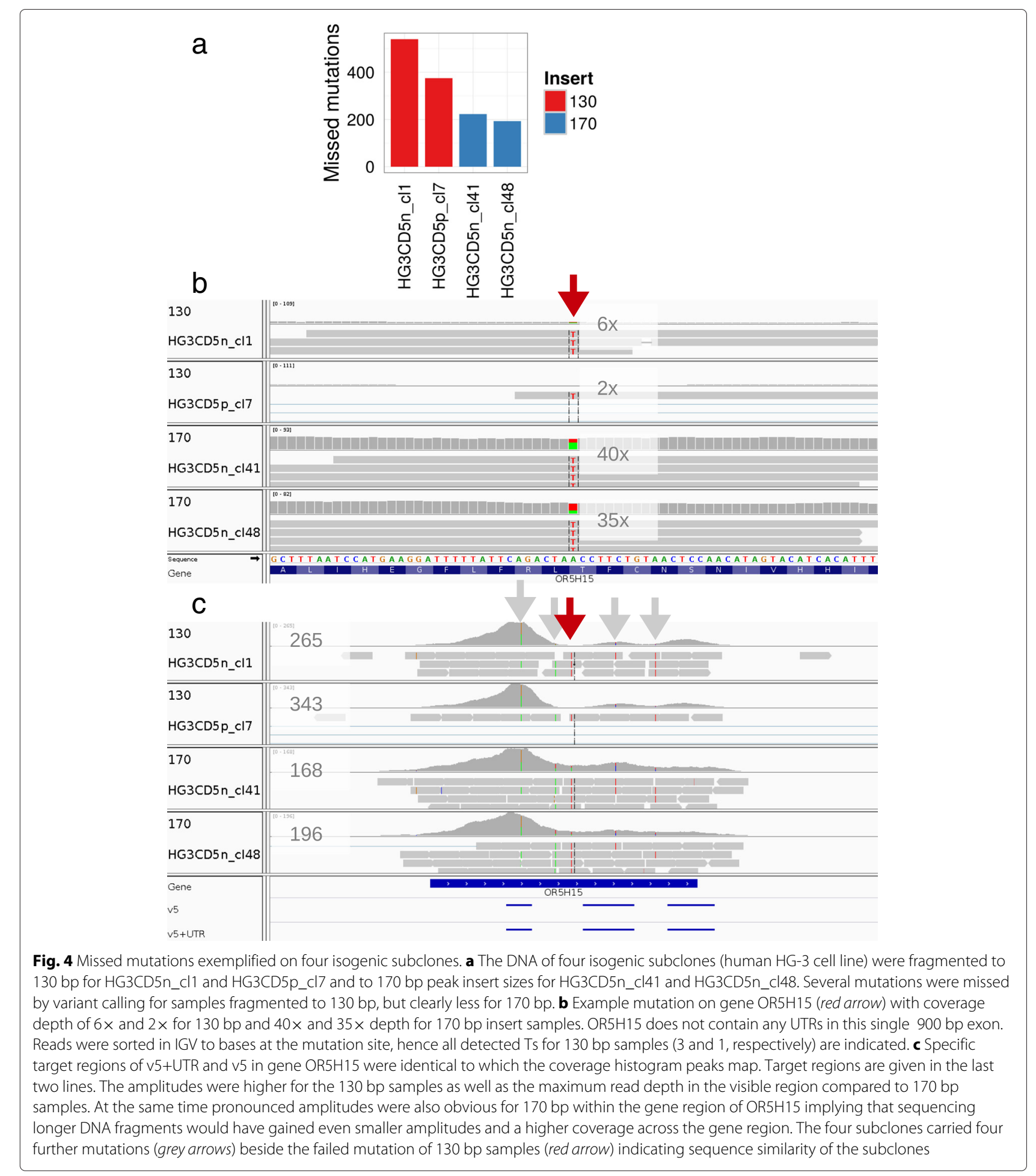

Agilent SureSelect All Exon v5 (S04380110) and Agilent SureSelect All Exon v5+UTR (S04380219). These files were converted to gencode v21 coordinates by the UCSC liftover tool and files (https://genome-store.ucsc.edu/). Insert sizes were determined by Picard tools. Graphs were created in the R/Bioconductor environment (http://www. bioconductor.org/) in particular applying ggplot2 [18]. For comparability the evenness score [14] served as metrics for the uniform coverage in the target regions.

Variant calling was conducted by VarScan 2 [19] to identify mutations in four subclones of the HG3 cell line with a minimum depth of $10 \times$ and $2 \times$ 
for an alternative allele. The four subclones were HG3CD5n_cl1 and HG3CD5p-cl7 for 130 bp peak insert sizes and HG3CD5n_cl41 and HG3CD5n_cl48 for 170 bp (see Table 1). Afterwards, the Variant Effect Predictor (release 77, http://www.ensembl.org/info/docs/tools/vep/ index.html) helped to concentrate on mutations in coding regions with severe consequences such as missense and stop gained effects.

Data were deposited at ArrayExpress under the accession number E-MTAB-4527.

\section{Abbreviations}

CNV, Copy number variation; DSMZ, Deutsche Sammlung von

Mikroorganismen und Zellkulturen, German Collection of Microorganisms and Cell Cultures; IGV, Integrative genomics viewer; UTR, Untranslated region; WES, Whole exome sequencing; WGS, Whole genome sequencing.

\section{Acknowledgements}

We thank Frank Klawonn and Roderick MacLeod for proofreading and advice. For technical assistance we would like to thank Margarete Zaborski. Last but not least we thank the two anonymous reviewers for improving this paper with many helpful and inspiring comments.

\section{Funding}

This work was supported by the Wilhelm-Sander-Stiftung [2013.061.1].

\section{Authors' contributions}

$\mathrm{CP}$ contributed to the experimental design, wrote the manuscript and performed all data preprocessing and analyses. BB discussed the concepts and proofread the manuscript. RG and SB discussed and supervised the sequencing procedure. HGD and SE conceived and proofread the manuscript. $\mathrm{HQ}$ assigned the experimental settings, provided data materials and wrote the manuscript. All authors have read and approved the manuscript.

\section{Competing interests}

The authors declare that they have no competing interests.

\section{Author details}

${ }^{1}$ Leibniz Institute DSMZ-German Collection of Microorganisms and Cell Cultures, Inhoffenstrasse 7B, 38124 Braunschweig, Germany. ${ }^{2}$ Helmholtz Centre for Infection Research, Inhoffenstrasse 7, 38124, Braunschweig, Germany.

Received: 22 October 2015 Accepted: 6 May 2016

Published online: 25 May 2016

\section{References}

1. Hodges E, Xuan Z, Balija V, Kramer M, Molla MN, Smith SW, et al. Genome-wide in situ exon capture for selective resequencing. Nat Genet. 2007;39(12):1522-7.

2. Shen T, Pajaro-Van de Stadt SH, Yeat NC, Lin JC. Clinical applications of next generation sequencing in cancer: from panels, to exomes, to genomes. Front Genet. 2015;6:215.

3. Bamshad MJ, Ng SB, Bigham AW, Tabor HK, Emond MJ, Nickerson DA, et al. Exome sequencing as a tool for Mendelian disease gene discovery. Nat Rev Genet. 2011;12(11):745-55.

4. Ng SB, Turner EH, Robertson PD, Flygare SD, Bigham AW, Lee C, et al. Targeted capture and massively parallel sequencing of 12 human exomes. Nature. 2009;461(7261):272-6.

5. Zhang G, Wang J, Yang J, Li W, Deng Y, Li J, et al. Comparison and evaluation of two exome capture kits and sequencing platforms for variant calling. BMC Genomics. 2015;16(1):581.

6. Nam JY, Kim NK, Kim SC, Joung JG, Xi R, Lee S, et al. Evaluation of somatic copy number estimation tools for whole-exome sequencing data. Brief Bioinform. 2015. pii: bbv055. [Epub ahead of print] PubMed PMID: 26. 210357.
7. Alkodsi A, Louhimo R, Hautaniemi S. Comparative analysis of methods for identifying somatic copy number alterations from deep sequencing data. Brief Bioinform. 2015;16(2):242-54.

8. Asan, Xu Y, Jiang H, Tyler-Smith C, Xue Y, Jiang T, et al. Comprehensive comparison of three commercial human whole-exome capture platforms. Genome Biol. 2011;12(9):R95.

9. Clark MJ, Chen R, Lam HY, Karczewski KJ, Chen R, Euskirchen G, et al. Performance comparison of exome DNA sequencing technologies. Nat Biotechnol. 2011;29(10):908-14.

10. Shigemizu D, Momozawa Y, Abe T, Morizono T, Boroevich KA, Takata S, et al. Performance comparison of four commercial human whole-exome capture platforms. Sci Rep. 2015;5:12742.

11. Meienberg J, Zerjavic K, Keller I, Okoniewski M, Patrignani A, Ludin K, et al. New insights into the performance of human whole-exome capture platforms. Nucleic Acids Res. 2015;43(11):e76.

12. Lelieveld SH, Spielmann M, Mundlos S, Veltman JA, Gilissen C. Comparison of Exome and Genome Sequencing Technologies for the Complete Capture of Protein-Coding Regions. Hum Mutat. 2015;36(8): 815-22.

13. Meynert AM, Ansari M, FitzPatrick DR, Taylor MS. Variant detection sensitivity and biases in whole genome and exome sequencing. BMC Bioinformatics. 2014;15:247.

14. Mokry M, Feitsma H, Nijman IJ, de Bruijn E, van der Zaag PJ, Guryev V, et al. Accurate SNP and mutation detection by targeted custom microarray-based genomic enrichment of short-fragment sequencing libraries. Nucleic Acids Res. 2010;38(10):e116.

15. Drexler HG. Guide to Leukemia-Lymphoma. Cell Lines, 2nd edition. Braunschweig; 2010.

16. Dobin A, Davis CA, Schlesinger F, Drenkow J, Zaleski C, Jha S, et al. STAR: ultrafast universal RNA-seq aligner. Bioinformatics. 2013;29(1):15-21.

17. Robinson JT, Thorvaldsdóttir H, Winckler W, Guttman M, Lander ES, Getz G, et al. Integrative genomics viewer. Nat Biotechnol. 2011:29(1):24-6.

18. Wickham H. ggplot2: elegant graphics for data analysis. New York: Springer; 2009. http://had.co.nz/ggplot2/book.

19. Koboldt D, Zhang Q, Larson D, Shen D, McLellan M, Lin L, Miller C, Mardis E, Ding L, Wilson R. VarScan, 2. Somatic mutation and copy number alteration discovery in cancer by exome sequencing Genome Res. 2012;22(3):568-76.
Submit your next manuscript to BioMed Central and we will help you at every step:

- We accept pre-submission inquiries

- Our selector tool helps you to find the most relevant journal

- We provide round the clock customer support

- Convenient online submission

- Thorough peer review

- Inclusion in PubMed and all major indexing services

- Maximum visibility for your research

Submit your manuscript at www.biomedcentral.com/submit
) Biomed Central 